\title{
Un univers « kafkaïen » aujourd'hui ?
}

\section{Gérald Berthoud}

\section{OpenEdition \\ Journals}

Édition électronique

URL : http://journals.openedition.org/ress/364

DOI : $10.4000 /$ ress.364

ISSN : 1663-4446

\section{Éditeur}

Librairie Droz

\section{Édition imprimée}

Date de publication : 1 janvier 2006

Pagination : 13-26

ISBN : 2-600-00959-0

ISSN : 0048-8046

Référence électronique

Gérald Berthoud, « Un univers « kafkaïen » aujourd'hui ? », Revue européenne des sciences sociales [En ligne], XLIV-133 | 2006, mis en ligne le 04 novembre 2009, consulté le 19 avril 2019. URL : http:// journals.openedition.org/ress/364; DOI : 10.4000/ress.364 


\section{Gérald BERTHOUD}

\section{UN UNIVERS «KAFKAÏEN» AUJOURD'HUI?}

A suivre les nombreux commentaires sur l'œuvre de Franz Kafka (18831924), celui-ci présente, dans ses romans et dans ses très brefs récits, des personnages enfermés dans des circonstances angoissantes, renvoyant aux figures de voies sans issue ou du labyrinthe (voir Le Bras 2000). Aussi l'adjectif «kafkaïen» est-il couramment employé pour qualifier «des situations absurdes, oppressives, ou sans issue de la vie moderne» (Raboin 1973: 7) et pour se référer à l'aspect incompréhensible et arbitraire du pouvoir pour ceux «d'en bas », jusqu'à évoquer l'horizon d'une société totalitaire'.

Mais pour qui veut dépasser cet usage banalisé d'une vague évocation des écrits de Kafka, les difficultés de lecture d'une œuvre aussi fragmentée sont considérables, en raison même des multiples interprétations possibles ${ }^{2}$. A moins d'en rester à un simple commentaire, la voie à suivre pour dégager la portée symbolique d'une telle œuvre est malaisée. Le mode d'interprétation de la réalité humaine et sociale par la fiction obéit à des exigences propres. Ce qui ne manque pas de poser de sérieux problèmes, quand il s'agit de traduire le message d'un roman ou d'un bref récit dans un langage de nature scientifique. Certes, l'objectif de l'activité littéraire et des savoirs argumentés sur l'homme et la société est semblable. L'une comme les autres partent d'une interrogation sur le monde pour en dévoiler des réalités obscurcies par des idées et des valeurs trompeuses, ou, tout au moins, illusoires. Tous tentent de s'imposer un difficile travail de déchiffrement. Mais les créations littéraires, au contraire des sciences sociales enfermées dans les limites étroites d'une quête infinie de scientificité, peuvent mieux présenter les actions humaines dans leur complexité, leur ambivalence, ou encore leurs paradoxes. La recherche de la vérité, propre à la démarche scientifique, tend à écarter les effets déstabilisateurs de toute interrogation fondamentale et à produire un savoir qui ne laisse guère de place aux doutes. L'œuvre littéraire peut ainsi mettre en évidence, dans l'expérience humaine, ce qui passe le plus souvent pour des «restes » pour les tenants d'une démarche scientifique conforme ${ }^{3}$. Aussi

Pour éviter les confusions et les malentendus, plusieurs auteurs francophones font la distinction entre les études «kafkéennes», ou l'œuvre «kafkéenne» et l'adjectif «kafkaïen» devenu un lieu commun.

2 «On comprend toujours plus ou toujours moins qu'il ne faut. La vraie lecture reste impossible. Celui qui lit Kafka est donc forcément transformé en menteur, et pas tout à fait en menteur » (Blanchot 1981: 66).

Sur l'apport spécifique des œuvres romanesques pour une pleine compréhension de la condition humaine, voir Kundera (2005). 
les créations inscrites dans l'ordre de la fiction devraient permettre de mettre en perspective les recherches scientifiques sur l'homme et la société, pour en dégager leurs limites. Certes, toute création littéraire est liée à une prise de conscience aiguë de la propre subjectivité de son auteur et de la réalité de son époque.

Aussi, avant même de tirer les leçons possibles d'une lecture attentive des écrits de Kafka, il importe de se demander quel lien est-il nécessaire d'établir avec son expérience personnelle, ou encore quelle part de son intériorité se retrouve dans ses écrits. Qu'il suffise de relever l'importance accordée par de nombreux auteurs aussi bien aux rapports conflictuels avec son père, cette première incarnation de la Loi et sa qualité de membre marginal de la minorité juive de Prague ${ }^{4}$. Mais au-delà même de sa personnalité, Kafka a vécu dans un contexte qui permet de comprendre à la fois la portée et les limites d'une œuvre nécessairement située et datée. Pour l'essentiel, elle s'inscrit dans la phase ultime de l'empire austrohongrois. Ce dernier se caractérise, entre autres, par une bureaucratie envahissante. Plus particulièrement dans la ville de Prague après 1880, dans laquelle Kafka passa sa vie, «la loi faisait figure d'oppresseur, et s'y soumettre revenait à se rendre complice de la destruction du monde» (Johnston $1985: 460$ ). Kafka avait une conscience aiguë de ces pesanteurs bureaucratiques (voir Angel 1962), en raison même de ses études de droit couronnées par un doctorat (1906), mais aussi de ses activités dans le domaine des assurances (1907-1923). Une voie académique et professionnelle qui portait tout naturellement à envisager les problèmes des êtres humains et de la société sous l'angle de la Loi, dans son sens le plus général ${ }^{5}$.

Cette expérience particulière n'en a pas moins une signification universelle, propre à interpeller le lecteur d'aujourd'hui. C'est dire que lire Kafka devrait permettre une interrogation renouvelée sur des aspects trop souvent occultés du monde actuel ${ }^{6}$. Il s'agit ici de s'engager dans une telle direction et de mettre à l'épreuve les possibilités de dévoilement d'une telle démarche, à partir du très bref récit intitulé «Devant la loi $»^{7}$. Celui-ci trouverait sa source d'inspiration dans la tradition talmudique. En effet, selon un récit rapporté par Origène (vers 185vers 254), «les Ecritures Saintes ressemblaient à une grande maison avec beaucoup, beaucoup de pièces; devant chaque pièce se trouve une clé, mais ce n'est

${ }^{4}$ Pour Hannah Arendt, Kafka appartenait à cette catégorie de Juifs qualifiés de «parias conscients » (1987: 74 et 205 et suiv.).

5 Dans le sens, entre autres, de ce qui est posé, institué ou établi et, en conséquence, de ce qui lie. Voir, par exemple, le terme allemand «Gesetz». La Loi peut ainsi être vue comme ordre du monde, à la fois comme institution qui prescrit et donc sanctionne et comme une prétendue nécessité naturelle.

- Cette portée symbolique de l'œuvre de Kafka n'est pas admise par tout le monde. Pour l'écrivain hongrois Imre Kertesz, prix Nobel de littérature en 2002 et auteur, en particulier du récit «Le procès-verbal »(2005), «Kafka est un réaliste typique de la Mitteleuropa » et son œuvre «traduit[...] la quintessence d'une vision du monde est-européenne» (Le Monde 10 juin 2005). Au contraire, pour Roberto Calasso, auteur, entre autres, d'un essai littéraire sur Kafka (voir 2005), les textes de ce dernier ne seraient pas des paraboles et aucune interprétation symbolique ne serait acceptable (voir Le Monde 3 juin 2005).

7 Ce texte a été publié par Kafka en 1919 et se retrouve sans titre, mais accompagné d'une exégèse relativement longue, à la fin du roman posthume «Le procès » (Kafka 1976: 453-461). Pour la première publication en français de «Devant la loi », voir Kafka (1929). 
pas la bonne. Les clés de toutes les pièces ont été échangées, et il faut [...] trouver les bonnes clés qui ouvriront les pièces » (voir Scholem 1980: 20). Une évidente ressemblance apparaît entre ces deux récits. Dans son exégèse, Kafka se réfère aussi «aux récits qui précèdent la Loi », ou au «texte de l'Ecriture (1976: 453 et $455)^{8}$. Mais une différence majeure doit être immédiatement relevée. Avec le premier récit, l'espoir de retrouver la «révélation» n'est pas vain. Les «clés » sont disponibles et malgré la difficulté de la tâche, il suffirait de trouver celle qui devrait ouvrir une porte précise. Chez Kafka, il n'y a tout simplement pas de clé pour une porte qui est toujours ouverte (voir Cacciari 1990: 73 et suiv.). C'est dire que le travail d'interprétation se heurte à d'évidents obstacles. Malgré le recours à l'exégèse, les lectures possibles sont nombreuses, au point d'avoir l'impression d'être confronté à un texte dont le sens serait proprement incompréhensible. Ce qui ferait la part belle à la subjectivité des lecteurs. Pourtant suivre une telle voie reviendrait à se faciliter la tâche, en esquivant la difficulté de s'interroger à la fois sur la nature du récit de Kafka et sur la relation qui peut s'établir selon le type de lecteur.

«Devant la loi» est présenté parfois comme une allégorie, mais le plus souvent comme une parabole. Dans un cas comme dans l'autre, ce récit devrait être porteur d'une signification et renvoyer à des principes moraux, comme l'attestent les paraboles inclues dans les trois Evangiles selon Saint Matthieu, Saint Marc et Saint Luc. Dans l'hypothèse d'une allégorie, chaque composante du récit, c'est-à-dire le gardien, l' «homme de la campagne» et la Loi devraient constituer des symboles bien identifiés se rapportant à des éléments clairement définis dans la réalité sociale et culturelle. Avec la parabole, prise dans un sens large, le procédé métaphorique est plus souple. En particulier, l'enseignement à tirer d'un tel récit est souvent très difficile à saisir. Le texte de Kafka est de ce point de vue tout à fait exemplaire. Si nous devons y voir une parabole, cette dernière ne contient aucune leçon. Quelle position le lecteur doit-il alors adopter face à un récit dépourvu apparemment de toute signification claire? Faut-il penser qu'une lecture attentive de l'exégèse permettrait d'éclairer le lecteur, ou tout au moins de le sortir de son embarras? Rien n'est moins sûr. D'abord parce que les deux protagonistes du dialogue dans la cathédrale, l'abbé et K., s'identifient chacun aussi bien au gardien, comme cet agent subalterne au service de la Loi, qu'à l' «homme de la campagne». Mais surtout l'abbé, à plusieurs reprises, se contente de rapporter les diverses opinions attribuées à des exégètes ou des commentateurs (voir Kafka 1976: 456-459). Autant dire que l'exégèse ne fournit pas les clefs pour comprendre la dimension symbolique du récit. Au contraire, elle tend à le rendre à proprement parler illisible. Ou tout au moins à multiplier les déclarations interprétatives, au point d'enfermer le lecteur dans une saisie immédiate et autoréférentielle du texte (voir Derrida $1985: 128-9$ ).

Comment alors se situer par rapport à un tel récit, dont l'exégèse ne fait qu'obscurcir le sens possible? Nul doute que le lecteur pourrait aisément verser dans une sur-interprétation au gré de son imagination, multipliant ainsi, de manière spéculative, les significations cachées du récit. De toute évidence, tenter

«Pour Kafka, la pensée de la loi, avant de relever de son métier de juriste, est liée à la quête de son identité juive» (voir Para 2002: 131). 
de savoir ce que signifient l'un ou l'autre texte de Kafka et plus encore l'ensemble de son œuvre est malaisé. Certes ce travail d'interprétation passe nécessairement par la manière dont la réalité est conçue par le lecteur.

De manière simplifiée, deux niveaux de lecture sont possibles. Tout d'abord, à suivre de nombreux représentants des études «kafkéennes», la portée symbolique de récits comme «Devant la loi » se ramène essentiellement à mettre en évidence la nature foncièrement angoissée de l'être humain. Mais pour d'autres auteurs, la lecture privilégiée porte rigoureusement sur le rapport à la Loi'.

\section{UNE EXISTENCE HUMAINE ANGOISSÉE?}

Pour de nombreux auteurs, intéressés, d'une manière ou d'une autre, à l'œuvre de Kafka, les personnages présentés par ce dernier sont l'objet d'une analyse proprement psychologique. Une série de termes plus ou moins proches permettent de constituer une sorte de nébuleuse, propre à évoquer la condition incertaine de l'être humain, ou encore la figure proprement kafkaïenne. Telles sont les notions de misère morale, d'angoisse, d'anxiété. La vie humaine serait une série d'échecs, entraînant chaque fois un sentiment profond de frustration et une impression de la futilité de toute action. Dans l'histoire de vie de l'«homme de la campagne», à peine esquissée, c'est l'absence de tout espoir, qui constituerait, à suivre quelques commentateurs, le message de la parabole «Devant la Loi ${ }^{10}$. Cette attente, pour laquelle les résultats désirés sont toujours remis à plus tard, tendrait à confirmer qu' «il n'y a de définitif que la souffrance» (Kafka dans Derrida 1985: 139). C'est dire que pour chacun tout semble joué à l'avance. Les uns, comme l' «homme de la campagne », s'identifient à des désirs inassouvis, alors que les autres, comme le gardien, ne sont guère plus que des obstacles insurmontables. Dans une telle confrontation entre individus isolés et réduits à des fonctions relativement bien définies, la Loi perd toute consistance. Seule semble entrer en ligne de compte une situation en quelque sorte expérimentale pour mettre pleinement en évidence l'insatisfaction foncière constitutive de la condition humaine. L'être humain, dans sa nature même, serait-il condamné à attendre quelque chose qui n'arriverait jamais? Cette vision de l'être humain d'un pessimisme extrême peut être relativisée. Ne faudrait-il pas voir chez Kafka un effort pour aborder le thème universel de la vie et de la mort? La condition humaine s'inscrit dans cette tension majeure, qui se

\footnotetext{
${ }^{9}$ De manière plus exigeante et plus minutieuse, les interprétations de l'œuvre de Kafka sont multiples. Elles portent, entre autres, sur une dimension métaphysique, sur un «en deçà de la vie », sur la condition misérable de l'homme, sur un refus de l'absurde et de l'arbitraire, sur la nécessité de donner un sens à la vie humaine contre toute forme de désespoir et sur bien d'autres thèmes encore. Mais pour de nombreux commentateurs de Kafka, une telle fragmentation interprétative est mise radicalement en question. Il s'agirait alors «d'abandonner le dogmatisme et le schématisme de l'approche thématique pour redonner aux récits de Kafka leur force interrogative et suggestive, et leur valeur de témoignage» (Tabery 1991: 11). Sur le plan quantitatif, en 1961 déjà, les travaux sur Kafka se montaient à plus de cinq mille titres (voir Raboin 1973: 7)

${ }^{10}$ Voir, par exemple, Herbert Deinert, «Kafka's parable Before the Law» (The Germanic Review, May 1964), repris, avec un «addendum», sur l'Internet: http://www.people.cornell.edu/pages/hd11/ BeforeTheLaw.html (consultation 17.03.2005).
} 
retrouve dans toutes les sociétés, selon des modalités très variables. L'homme se heurte à la finitude propre à tout être vivant, mais partout et toujours il imagine un univers de sens pour ne pas sombrer dans l'absurde. L' « homme de la campagne», par exemple, malgré son attente et ses échecs répétés, ne semble pas verser dans le désespoir.

\section{LE RAPPORT À LA LOI}

Mais en rester à ce seul niveau de la subjectivité reviendrait à omettre la portée sociale et politique de l'œuvre de Kafka. Pour ce dernier, les divers personnages frustrés de ses romans et de ses récits ne sont concevables que dans leurs relations nécessaires avec des lois et des institutions, qui leur imposent un mode d'organisation et qui donnent, ou devraient donner, un sens à leur vie. Il serait bien insuffisant d'en rester à une description et à une analyse des motivations humaines, sans aucune considération du contexte institutionnel. La lecture privilégiée ici, sans minimiser la portée des récits de Kafka pour symboliser la précarité, l'incertitude et le mal-être constitutifs de l'expérience humaine individuelle, est celle qui porte sur une interprétation du point de vue du «vivre ensemble». En d'autres termes, le thème du rapport à la Loi devrait permettre d'aborder, une fois de plus, l'interrogation fondamentale de ce qu'être humain veut dire. Ce qui implique, entre autres, de se rapporter tout à la fois aux idées de loi, d'institution, d'ordre, de valeur, ou encore de sens. Mais avant tout il importe de savoir quel sens donner à la notion de Loi. Très rigoureusement, il n'est guère possible de définir ce que Kafka entendait par Loi ou à quel type de loi il se référait. La Loi peut s'objectiver dans un texte, dans des paroles, dans une personne, mais aussi dans des bâtiments. Mais pour une pleine compréhension du récit de Kafka, peut-être est-il vain d'insister sur ces formes d'extériorisation de la Loi. Sans nul doute, Kafka ne porte pas son attention sur une loi particulière; il ne se réfère pas exclusivement à la loi au sens religieux, moral, juridique, ou plus largement politique, ni au thème de la «loi naturelle», de la «loi juive», ou encore de la «loi paternelle». Aussi partons de l'hypothèse qu'il s'agit de l'idée de la Loi dans le sens le plus abstrait ${ }^{11}$.

La Loi ainsi entendue, comme source de toute loi positive ou de toute institution spécifique, permet d'instaurer et d'organiser un espace social. Mais cet univers de sens partagé n'est acceptable que pour autant qu'il s'articule avec le thème de la liberté. Il y a là une manière parmi d'autres d'exprimer une vérité anthropologique, celle d'une tension universelle entre obligation et liberté Certes ces deux notions ne sont pas pleinement satisfaisantes. L'idée de liberté tend à être vue comme le privilège de la modernité. A moins d'accepter qu'elle «admet des contenus différents » (Lévi-Strauss 1983: 373) ${ }^{12}$. Avec la notion d'obligation, les

11 «Dans le récit de Kafka, on ne sait pas de quelle espèce de loi il s'agit, celle de la morale, du droit ou de la politique, voire de la nature, etc. Ce qui reste invisible et caché en chaque loi, on peut donc supposer que c'est la loi elle-même, ce qui fait que ces lois sont des lois» (Derrida 1985: 109-110).

${ }_{12}$ «Qu'il n'y ait pas d'opposition entre la contrainte et la liberté, qu'au contraire elles s'épaulent toute liberté s'exerçant pour tourner ou surmonter une contrainte, et toute contrainte présentant des fissures ou des points de moindre résistance[...] - rien ne peut mieux, sans doute, dissiper l'illusion contemporaine que la liberté ne supporte pas d'entraves[...]: illusion qui n'est certes pas la cause, mais où l'on peut voir un aspect significatif de la crise que traverse aujourd'hui l'Occident » (Lévi-Strauss 1983: 17). 
difficultés sémantiques ne sont pas moindres. Est-elle, par exemple, un simple synonyme de contrainte? Pour nombre d'auteurs, ces deux termes sont interchangeables. Une différenciation serait pourtant nécessaire. En fait cette tension est le plus souvent ramenée à une opposition générale et vague entre un pôle individuel et un pôle collectif. Dans cette perspective, toute société, marquée par une recherche permanente d'équilibre, devrait être organisée de manière à ce que chacun puisse affirmer son identité subjective et être reconnu comme un membre à part entière. Cette tension primordiale, constitutive de toute vie proprement humaine, fait que toute relation, à commencer par le rapport à soi-même, implique nécessairement un entre-deux, une médiation, ou encore un tiers. Ces derniers sont autant de symboles de valeurs partagées, ou d'institutions propres à une collectivité donnée, en mesure d'unir et de rassembler ses membres malgré les évidents conflits ${ }^{13}$. Sauf à tomber dans l'immédiateté de formes fusionnelles ou violentes de l'indifférenciation, tout rapport à autrui, même le plus proche, n'est envisageable que sous l'angle de la «bonne distance»(Ost 2004: 344). Toute relation intersubjective est donc instituée. Certes, entre un tiers comme le don, dans une perspective maussienne, comme forme matricielle de toute socialité et un tiers en surplomb comme l'Etat et son mode d'imposition pour organiser la société, les différences sont énormes. Mais dans tous les cas, les relations humaines sont fondamentalement triadiques ${ }^{14}$ et supposent, pour se maintenir à travers le temps, l'existence de symboles. Dans cette perspective, la nécessité de la Loi et sa force organisatrice faite d'interdits, propres à rendre visibles les croyances fondatrices, s'imposent pour contenir, au double sens du terme, toute conduite rigidement individualiste. La Loi rend la société possible, par la régulation des relations humaines.

La question se pose alors de l'origine de cet événement premier, certes fictif, mais essentiel pour légitimer un ordre social et ses multiples lois. C'est dire que toute société doit s'envisager dans une temporalité dans laquelle le passé n'est pas tout simplement occulté. Mais ce passé ainsi inventé n'échappe pas aux effets de toute forme d'imagination, propre à faire naître une réalité sociale à partir de croyances susceptibles d'un usage partisan et de manipulations, ou encore de domination symbolique. Aussi dans la tradition de toute société, l'imaginaire instituant établit un lien ambigu entre les membres vivants et une altérité radicale, ou une entité supérieure de nature religieuse ou politico-religieuse. Même pour les sociétés dites modernes, cette relation verticale avec une forme ou l'autre de l'invisible n'a pas disparu, malgré les dénégations des défenseurs d'une modernité en rupture radicale avec toute forme de tradition. En bref, toute société est instituée sur la base d'un donné inconditionnel. C'est dire qu'il y a toujours du « déjà là ». En somme, un rapport à la loi de nature démocratique devrait permettre le maintien d'un équilibre toujours instable entre les deux pôles de la liberté et de l'obligation.

${ }^{13}$ La notion d'institution est prise ici dans un sens large. A suivre Mauss, il s'agit de «règles publiques d'action et de pensée» (1968: 25). Dans cette perspective, «le langage est la grande institution - l'institution des institutions - qui nous a chacun toujours précédé » (Ricœur 1985: 400).

${ }^{14}$ Sur la notion de triade, voir Pierce (1965: 253-56). Un tel triangle constitutif de toute socialité s'exprime dans le jeu des trois pronoms «je», «tu» et «il» (voir Benveniste 1966: 225-236). 
Mais une question redoutable ne manque pas de se poser immédiatement. Jusqu'où le désir de liberté peut-il se manifester sans mettre en danger la Loi? Pour un lecteur centré sur la composante sociale et politique de l'être humain, la Loi très immédiatement apparaît sous une forme extériorisée ou objectivée dans un texte comme la Constitution, mais aussi dans de nombreux mythes ou récits fondateurs des sociétés modernes et traditionnelles. Se pose immédiatement la question de savoir jusqu'à quel point ces récits sont intériorisés. L'enjeu est alors celui de savoir si la Loi est effectivement une partie tierce propre à unir une pluralité d'individus pour former un «nous». Ou si, au contraire, la Loi est vécue comme une stricte extériorité contraignante et comme l'affirmation tangible de ce qui est interdit. La Loi réduite ainsi à l'interdit équivaut à la figure de la mort, représentative des multiples formes de totalitarisme.

Kafka nous entraîne dans une vision du monde dichotomique entre une fraction dont la domination peut aller jusqu'au pouvoir absolu et une majorité dont la soumission peut être totale. Certes, le bref récit «Devant la loi» ne se rapporte pas à proprement parler à un pouvoir totalitaire. Néanmoins il illustre de manière exemplaire les dérives inévitables de toutes les sociétés travaillées par des forces de désymbolisation (voir Garapon 1997 :289). Il montre avec force la permanence d'un conflit entre une loi insaisissable et une vaine recherche de la vérité et de la justice. Il nous laisse entendre que la Loi est cachée, voilée, masquée, perdue et donc lointaine. Une succession de nuances pour dire que la Loi, tout en étant inaccessible, impose, à travers ses représentants, une obéissance sans concession. Une tendance qui peut facilement se transformer en un univers soumis à la violence d'une nécessité indiscutable et implacable. Dans l'exégèse qui suit la parabole «Devant la loi », l'abbé, dans son dialogue avec K., le personnage principal du roman «Le Procès », insiste sur le fait que le gardien est un «serviteur de la Loi » et comme tel «il appartient à la Loi». Aussi «douter de la dignité du gardien, ce serait douter de la Loi». Une manière de confirmer la supériorité du gardien qui «échappe donc au jugement humain ». Mais, pour K., accepter une telle vision reviendrait à «croire tout ce que dit le gardien». Et l'abbé de rétorquer alors qu' « on n'est pas obligé de croire vrai tout ce qu'il dit, il suffit qu' on le tienne pour nécessaire». Le dialogue prend fin avec ce commentaire désabusé: «triste opinion, elle élèverait le mensonge à la hauteur d'une règle du monde » (Kafka 1976: 459-60).

Une véritable «loi de nécessité» (Ost 2004: 339) imposerait ainsi une division radicale entre ceux qui doivent se soumettre sans condition et ceux qui apparaissent comme l'incarnation d'une telle loi, détournée à leur avantage. Poussée jusqu'à son extrême limite, la soumission peut aboutir à la perte de ce qui est proprement humain. Telle est chez Kafka la forte image du chien, ou le recours à d'autres métamorphoses pour signifier une vie humaine abjecte et aliénante. A partir de ce pessimisme outrancier, faudrait-il penser que chez Kafka, l'être humain, représenté, par exemple, par l' «homme de la campagne» est voué, sa vie durant, à attendre en vain? En fait, les personnages kafkéens sont engagés dans une recherche de la vérité et de la justice, ou tout au moins d'une signification existentielle. Une quête qui ne s'abîme pas dans la désespérance, malgré de nombreux signes propres à traduire l'idée d'une barrière infranchissable et l'expérience répétée de l'échec face à des obstacles toujours renouvelés. Une telle lueur d'espoir est bien attestée dans l'exégèse. Elle est esquissée par l'abbé 
comme une autre thèse qui complexifie celle de la «loi de nécessité». Ainsi le gardien se tromperait à la fois sur la Loi, sur lui-même et sur l'«homme de la campagne». Ce dernier comme «homme libre» est «supérieur à l'homme lié» (Kafka 1976: 458). Comme tel cet être doit résister, ne pas se laisser faire, se battre pour affirmer sa condition d'être humain, même si l'espoir de l'emporter est faible. Ou encore, tout faire pour ne pas être traité comme un chien, ou ne pas être enfermé dans «l'univers des choses» (Henel dans Raboin 1973: 84; voir aussi Gabel 1953: 955). Etre dominé ou être instrumentalisé, c'est devenir insignifiant, en faisant l'expérience de la honte et de la souffrance; c'est encore perdre tout estime de soi et toute reconnaissance d'autrui.

Les deux thèses, ébauchées dans l'exégèse, par leur juxtaposition même, traduisent un profond malaise au sujet du pouvoir et de la liberté individuelle. La loi, qui tend à être perçue comme une dualité foncière, est la garantie même de la liberté pour les uns et pour les autres; mais elle est aussi une instance centrée essentiellement sur la surveillance et le contrôle, pour un fonctionnement efficace de la société. Mais cette coexistence du pouvoir et de la liberté, vus abstraitement comme deux éléments radicalement opposés, sont en fait indissociables et inscrits dans une relation marquée par une ambiguïté intrinsèque. Nous retrouvons ici les difficultés d'interprétation de l'exégèse affirmant successivement la supériorité du gardien comme le détenteur de la Loi et la supériorité de l'homme libre. Pris dans une telle ambiguiité, l'abbé est dans l'incertitude; il est littéralement «poussé de côté et d'autre» sans trancher. Mais pouvait-il faire autrement? Liberté et obligation, dans le sens de ce qui est exigé par la Loi, ne sont-elles pas, d'une manière ou d'une autre, constitutives d'une relation ambivalente et paradoxale partout et toujours? Mais ces deux thèses tendent à montrer que la tension entre l'affirmation d'un «je» comme sujet libre et la nécessité de la Loi pourvoyeuse de sens et condition d'un «nous» politique se transforme en une dichotomie. Les deux pôles de la relation, devenus en quelque sorte indépendants, sont portés ainsi à s'affirmer sans limites.

\section{ET AUJOURD'HUI?}

Ne sommes-nous pas pris dans un tel dédoublement? Qu'en est-il de «la force interrogative et suggestive» de «Devant la loi» pour penser notre situation actuelle dans l'ère dite informationnelle (voir Berthoud 2005: 375)? La condition de l'individu d'ajourd'hui peut-elle être comparée à celle de l'«homme de la campagne »? Peut-elle être comprise à partir des deux thèses de l'exégèse? Plus largement, quelle leçon un lecteur peut-il retirer de ce bref texte de Kafka? Quel regard permet-il de porter sur le monde actuel? Ou encore, en quoi éclaire-t-il des enjeux majeurs, à la fois scientifiques et politiques? La difficulté, pour pouvoir pleinement répondre à de telles questions, est bien sûr de concilier les exigences d'un texte à prétention scientifique avec les principes propres à la création littéraire. La lecture de «Devant la loi » et sa mise en perspective dans le contexte de l'œuvre de Kafka suggèrent diverses pistes pour éclairer des aspects majeurs du monde actuel. Toutefois, en se référant explicitement aux deux thèses avancées dans l'exégèse, celles de la supériorité du «gardien», ou, au contraire de l'«homme libre », l'éventail de questions qu'il est possible de poser, pour parvenir à une certaine intelligibilité du temps présent, se ferme considérablement. Posons, 
à titre d'hypothèse, que la dualité foncière de la Loi, mise en évidence chez Kafka, tend à être perçue et vécue comme une revendication sans partage de la liberté et du choix, jusqu'à se muer en une illusion individualiste, de type libéral ou libertaire, pour laquelle rien ne devrait faire obstacle. Une exigence inconditionnelle de liberté qui se heurte à de nombreuses formes de pouvoir, portées à exercer sur l'être humain une force contraignante et à se situer au-dessus de tout. Cette croyance moderniste pose donc que le sujet individuel doit tendre vers une liberté accrue, face à des contraintes extérieures, qui sont sociales et politiques, mais aussi physiques et biologiques.

Mais soumis à l'emprise toujours plus forte de l'instrumental et du fonctionnel, tout particulièrement dans les domaines de l'économie et des technosciences largement vus comme une fin en soi, l'«homme libre» fait toujours davantage l'expérience d'une «crise de sens» ou d'une «crise d'identité». Sa revendication d'être une personne reconnue dans sa subjectivité, jusqu'à voir dans sa liberté une propriété de soi-même et de verser dans le fantasme de faire de son désir la Loi, bute nécessairement sur l'omniprésence toujours plus effective de la puissance pure portée à transgresser toute limite ${ }^{15}$. Tels sont d'abord les champs technoscientifique et économique, devenus, dans une large mesure, un monde extérieur à la perception et au vécu de l'«homme libre». Un monde qui impose toujours davantage un système de régulation automatique ou spontanée (voir Garapon 1997: 284) ${ }^{16}$. Une pareille situation ne manque pas de produire des effets pervers multiples. En particulier, elle entraîne une simplification trompeuse, qui pousse l'individu à s'opposer à un collectif contraignant, mais mal défini et généralement qualifié de société ${ }^{17}$. Cette dichotomie pose, entre autres, que la «société » serait une réalité extérieure, ou encore étrangère aux individus. La Loi ne peut alors apparaître que sous la forme d'une force brutale et arbitraire, détenue souvent par des représentants vénaux et pervertis. Toute obéissance à de telles «autorités», proprement incompréhensibles, tend à reposer sur une profonde méconnaissance.

\section{DE LA PUISSANCE TECHNOSCIENTIFIQUE ET ÉCONOMIQUE}

Aujourd'hui, à des degrés variables et selon des modalités multiples, la distance entre les individus ou les citoyens et ceux qui détiennent la puissance technoscientifique, économique et politique tend à s'accroître au point de consti-

\footnotetext{
${ }^{15}$ Pour le savoir technoscientifique, «la grande affaire n'est ni la vérité ni l'universalité, mais la puissance. La puissance au sens de domination, contrôle, maîtrise sans doute, mais aussi, et de plus en plus, au sens d'actualisation illimitée du possible par des pratiques manipulatrices et opératrices appliquées à une matière extraordinairement plastique qui inclut le vivant (et donc l'être humain)» (Hottois 1994: 150).

${ }^{16}$ Pour la tradition libérale, par exemple, «où l'intérêt commande, il ne faut pas de loi » (voir Larrère 1992:168). Une telle croyance renvoie bien sûr à l'idée de «l'harmonie naturelle des intérêts », ou à la notion de «main invisible».

${ }^{17}$ Parler de «société», c'est concevoir ce qui tient ensemble les membres d'une collectivité, malgré les différences et les conflits. Mais la représentation dominante aujourd'hui n'est-elle pas celle d'une fragmentation sociale, en insistant uniquement sur ce qui sépare individus et groupes?
} 
tuer une fracture sociale et culturelle. Une fracture repérable dans de multiples situations entre ceux qui prétendent parler et agir au nom de la Loi et qui exercent leur pouvoir sans partage et ceux qui la subissent. Crise de confiance donc entre ceux qui se croient les authentiques gardiens de la vérité, comme la seule voie pour parvenir au bien-être généralisé pour tous. Cette représentation dichotomique, entre un individu pur et l'inévitable réalité de contraintes objectives, a perdu une bonne partie de sa force à la suite de l'attentat du 11 septembre 2001 à New York et Washington. Cette catastrophe mondialisée a produit partout, selon des modalités variables, une «demande sécuritaire» et plus généralement une inquiétude. Dans cet enjeu majeur entre liberté et sécurité, la promesse d'un monde à la fois plus sécurisé et plus libre serait fournie par un surcroît de moyens techniques toujours plus efficaces.

Nombres d'auteurs, sensibles aux problèmes que posent les technologies dites nouvelles pour les individus, restent rigoureusement enfermés dans les limites d'une vision dichotomique. "L'individu contemporain, ce serait l'individu déconnecté symboliquement et cognitivement du point de vue du tout, l'individu pour lequel il n'y a plus de sens à se placer du point de vue de l'ensemble» (Gauchet 2002: 254). Une tendance qui se vérifie tout particulièrement dans le débat actuel sur le thème devenu central de la «vie privée ${ }^{18}$. Une opinion très suivie voit dans les technologies de l'information et de la communication la clé pour une protection assurée de la sphère privée. Mais dans une «société d'individus », le maintien de l'ordre social passe de plus en plus par l'imposition de techniques de surveillance toujours plus efficaces. L'être humain, dans l'ensemble de ses activités comme citoyen, travailleur et consommateur, est de plus en plus catégorisé, en vue de satisfaire les exigences techniques des multiples bases de données ${ }^{19}$. Autant dire que cette valorisation de la sphère privée et ce repli sur ses propres affaires, d'une part et le développement accéléré des technologies de surveillance, d'autre part s'inscrivent dans une «boucle de rétroaction perverse» (Lyon 2001: 21). Et surtout l'horizon d'une possible interconnexion effective des multiples bases de données, portant sur les individus et sur leurs actions, pourrait bien être celui d'une régulation proprement totalitaire des relations humaines ${ }^{20}$. Aujour-

${ }^{18}$ Pour Tocqueville, dans un chapitre de son ouvrage «De la démocratie en Amérique», intitulé «De l'individualisme dans les pays démocratiques», on peut lire: «L'individualisme est un sentiment réfléchi et paisible qui dispose chaque citoyen à s'isoler de la masse de ses semblables et à se retirer à l'écart avec sa famille et ses amis; de telle sorte que, après s'être ainsi créé une petite société à son usage, il abandonne volontiers la grande société à elle-même» (1961: 143).

19 «The convenience of the telephone or the credit card means that we seldom think twice about the fact that our calls and transactions are traceable and that others profit from using these data » (Lyon 2001:3).

${ }^{20}$ Dans un de ses derniers chapitres, avec le titre tout à fait explicite «Quelle espèce de despotisme les nations démocratiques ont à craindre », Tocqueville affirme: «l'espèce d'oppression dont les peuples démocratiques sont menacés ne ressemblera à rien de ce qui l'a précédée dans le monde[...]je vois une foule innombrable d'hommes semblables et égaux qui tournent sans repos sur eux-mêmes pour se procurer de petits et vulgaires plaisirs, dont ils emplissent leur âme » Et il poursuit: «cette sorte de servitude, réglée, douce et paisible[...], pourrait se combiner mieux qu'on ne l'imagine avec quelques-unes des formes extérieures de la liberté[...] Nos contemporains sont incessamment travaillés par deux passions ennemies: ils sentent le besoin d'être conduits et l'envie de rester libres »(1961: 433-34 et 435). 
d'hui déjà, l'insistance sur la vie privée est indissociable d'un conformisme social $^{21}$.

A suivre une croyance diffuse, les problèmes sociaux et politiques peuvent trouver une solution technoscientifique. Une technicisation progressive de la société peut laisser croire que la technologie pourrait assurer une régulation sociétale, en mesure de garantir un équilibre entre les revendications de liberté individuelle et la nécessité d'un ordre socio-politique. La présence toujours plus forte, voire envahissante, d'écrans dans les lieux publics et dans la sphère privée, et l'usage en voie de généralisation des cartes dites intelligentes sont autant de médiations électroniques, pour lier personnes et groupes sociaux dans un ensemble de réseaux. Ce processus peut donner l'impression que ce qui unit les individus, sans la moindre considération de limites géographiques et politiques, relève foncièrement d'une construction technoscientifique. Cette voie suppose une limitation considérable de toute action proprement politique, vue comme une ingérence inacceptable dans la vie privée. Cette revendication d'une forme d'individualisme s'inscrit dans la mouvance d'une modernité marquée par la toutepuissance d'un imaginaire portant sur «l'expansion illimitée de la 'maîtrise rationnelle'» (Castoriadis 1990: 17). Dans un tel contexte historique et culturel, les domaines conjoints de la technoscience et de l'économie occupent une place hégémonique, au point de donner un sens à un monde imposant de manière outrancière ouverture, flexibilité, dynamisme et valorisant tout ce qui suppose un changement en soi. Une façon de légitimer des valeurs vues comme essentielles pour une libération individuelle. Ces domaines tentent ainsi d'imposer une manière instituée d'être, de penser, de faire et d'agir, en mesure de soumettre à leur «loi » l'ensemble de la vie humaine.

\section{DU MALAISE DES SCIENCES SOCIALES}

Cette imposition d'une représentation aussi réductrice d'un monde proprement dépolitisé entraîne-t-elle une attitude irrémédiablement pessimiste? Faut-il accepter comme une fatalité historique la fragilité du «nous», de l'échelon local au niveau planétaire? Et surtout ne sommes-nous pas, dans nos activités scientifiques, une figure ambivalente, dans le sens d'être tout à la fois l'«homme de la campagne» et le «gardien» (voir Derrida 1985: 129 et 133)? Tous les deux ne sont-ils pas, chacun à leur manière, prisonniers d'une même vision du monde, des valeurs qu'elle suppose, des institutions qui lui donne forme et des pratiques qu'elle oriente ${ }^{22}$ ? Ces interrogations et bien d'autres encore traduisent l'indéniable malaise des sciences sociales aujourd'hui. Et pourtant le pessimisme intellectuel, caractéristique d'une large part des savoirs sur l'être humain et la société,

${ }^{21}$ Ne faudrait-il pas alors se demander s'il est encore possible de voir dans la valorisation de la vie privée une modalité de l'individualisme? Peut-être serait-il plus adéquat de parler de «privatisme» (voir Laville 2001 : 85).

${ }^{22}$ Kafka lui-même pourrait illustrer une même ambivalence. N'est-il pas à la fois celui qui passe une partie de ses nuits à écrire dans la perspective de ceux «d'en bas » et celui qui occupe, grâce à son doctorat en droit, une place relativement importante dans une compagnie d'assurances contre les accidents? 
n'élimine nullement une réflexion constructive. A commencer par la prise en considération d'un décalage, trop souvent occulté, entre l'évidence aveuglante des forces de transformations technoscientifiques et économiques et des manières traditionnelles toujours vivantes d'établir et de maintenir des relations avec autrui. Les diverses manifestations de la puissance ne vont pas sans des formes toujours renouvelées de résistance. Tout espoir n'est donc pas perdu. Loin s'en faut. Aussi n'est-il guère aisé de répondre à la question de savoir si le monde actuel pourrait être assimilé à un univers kafkaïen. Une première difficulté réside bien sûr dans l'usage généralisé et vague de cet adjectif. Il n'en reste pas moins que la force évocatrice de ce terme permet de penser la complexité de la condition humaine sous un éclairage renouvelé. L' «homme de la campagne», imaginé par Kafka, nous renvoie, entre autres, à la supériorité de l'homme libre qui veut connaître la Loi, c'est-à-dire disposer de repères pour pouvoir agir et donner un sens à sa vie. Ce que l' «homme de la campagne » cherche à connaître, ou à redécouvrir, c'est une Loi génératrice d'un sentiment d'appartenance. Ce mode institué d'être et d'agir devrait permettre d'établir un véritable barrage contre deux mouvements radicalement séparés et opposés, dans le prolongement de la double interprétation de l'abbé dans l'exégèse de «Devant la loi ». D'une part, la recherche de la puissance, caractérisée par l'illimitation et l'artificialisation et objectivée dans des actions scientifiques, techniques et économiques, mais aussi sociales et politiques. D'autre part, un individualisme exacerbé, apparenté à un relativisme postmoderne et légitimé, de manière paradoxale, par une sorte de «droit à l'indifférence» dans le sens d'une insensibilité ou d'une absence de considération pour les autres.

\section{Université de Lausanne}

\section{RÉFÉRENCES}

Angel, Pierre, 1962, «L'obsession bureaucratique chez Kafka». Etudes Germaniques 1: 1-13.

Arendt, Hannah, 1987, La tradition cachée. Le Juif comme paria. Paris: Bourgois (première publication en anglais 1946).

Benveniste, Emile, 1966, Problèmes de linguistique générale. Paris: Gallimard.

Berthoud, Gérald, 2005, «Pervasive Computing. Leading to Posthumanism?». Dans Société de l'information. Récits et réalité, G. Berthoud, A.Kündig et B.Sitter-Liver, eds. Fribourg: Academic Press (22 colloque de l'Académie suisse des sciences humaines et sociales), p. 373-386.

Blanchot, Maurice, 1981, De Kafka à Kafka. Paris: Gallimard.

Cacciari, Massimo, 1990, Icones de la loi. Paris: Christian Bourgois (première publication en italien 1985).

Calasso, Roberto, 2005, K. Paris: Gallimard (traduit de l'italien).

Castoriadis, Cornelius, 1990, «L'époque du conformisme généralisé». Dans Le monde morcelé. Paris: Seuil, p. 11-24.

Derrida, Jacques et al., 1985, La faculté de juger. Paris: Minuit (Colloque de Cerisy).

Gabel, Joseph, 1953, «Kafka, romancier de l'aliénation». Critique 78: 949-960.

Garapon, Antoine, 1997, Bien juger. Essai sur le rituel judiciaire. Paris: Odile Jacob.

Gauchet, Marcel, 2002, La démocratie contre elle-même. Paris: Gallimard.

Hottois, Gilbert, 1994, «Jeux de langage et pratiques technoscientifiques ». Dans Richard Rorty. Ambiguïtés et limites du postmodernisme, G. Hottois et M. Weyembergh, éds. Paris: Vrin, p. 139-180. 
Johnston, William M., 1985, L'esprit viennois. Une histoire intellectuelle et sociale 1848-1938. Paris:PUF (première publication 1972, Etat-Unis).

Kafka, Franz, 1929, «Bucéphale». La Nouvelle revue française 191: 205-211(incluant les textes suivants: 'Le nouvel avocat', 'Devant la loi', 'Un message impérial', 'Le plus proche village', 'Il tue son frère').

Kafka, Franz, 1976, Euvres complètes I. Paris: Gallimard.

Kundera, Milan, 2005, Le rideau. Paris: Gallimard.

Larrère, Catherine, 1992, L'invention de l'économie au XVIII siècle. Du droit naturel à la physiocratie. Paris: PUF.

Laville, Jean-Louis, 2001, «Travail: la nouvelle question politique». Revue du M.A..US.S. 18: 79-91.

Le Bras, Hervé, 2000, «La métaphore du labyrinthe chez Kafka». Revue européenne des sciences sociales 117: 159-171.

Lyon, David, 2001, Surveillance Society: Monitoring Everyday Life. Buckingham: Open University Press.

Lévi-Strauss, Claude, 1983, Le regard éloigné. Paris: Plon.

Mauss, Marcel, 1968, Euvres 1. Les fonctions sociales du sacré. Paris: Minuit.

Ost, François, 2004, «Kafka ou l'en deçà de la loi ». Dans Raconter la loi. Aux sources de l' imaginaire juridique. Paris: Odile Jacob, p. 337-405.

Para, Jean-Baptiste, 2002, «Kafka devant la loi. Entretien avec Dolf Oehler ». Europe : revue littéraire mensuelle, avril: 122-133.

Pierce, Charles Sanders, 1965, Collected Papers of Charles Sanders Pierce». Vol. I Principles of Philosophy. Cambridge, Mass.: The Belknap Press of Harvard University Press.

Raboin, Claudine, éd., 1973, Les critiques de notre temps et Kafka. Paris: Garnier.

Ricœur, Paul, 1985, Temps et récit 3. Le temps raconté. Paris: Seuil.

Scholem, Gershom G., 1980, La Kabbale et sa symbolique. Paris: Payot (première publication en allemand 1960).

Tabery, Françoise, 1991, Kafka en France, Essai de bibliographie annotée. Paris: Lettres Modernes.

Tocqueville, Alexis de, 1961, De la démocratie en Amérique II. Paris: Gallimard. 
